$\underline{\text { Preprint typeset in JHEP style. - HYPER VERSION }}$

LPT-ENS/01-38, hep-th/0112nnn

\title{
Gravitational couplings of orientifold planes
}

\author{
Pierre Henry-Labordère and Bernard Julia \\ Laboratoire de Physique Théorique de l'Ecole Normale Supérieure* \\ 24 rue Lhomond, 75231 Paris Cedex 05, France \\ phenry@lpt.ens.fr \\ bernard.julia@lpt.ens.fr
}

\begin{abstract}
We reanalyse the gravitational couplings of the perturbative orientifold planes $\mathrm{Op}^{-}, \mathrm{Op}^{+}$(and D-branes). We first compute their $\mathrm{D}_{-1}$ instantonic corrections for $p=3$. Then, by using U-dualities, we obtain the Wess-Zumino terms of orientifolds with RR flux for $p \leq 5$. The expressions for the effective actions can be partially checked via M-theory. We point out a previous oversimplification and we show in fact that the difficulty still stands in the way of the full computation of 7 Brane instanton corrections.
\end{abstract}

KEYwords: Orientifolds, D-instanton corrections, M-theory.

*Unité mixte du CNRS et de l'Ecole Normale Supérieure, UMR 8549. 


\section{Introduction}

Four kinds of orientifold p-planes with sixteen supersymmetry charges exist: $\mathrm{Op}^{-}$, $O p^{+}$and for $(p \leq 5) \widetilde{O p^{-}}$or $\widetilde{O p^{+}}$[1, 2]. Only $O p^{-}$and $O p^{+}$can be described perturbatively (in the string coupling) as the fixed planes of the orientifold projection $\Omega$ which acts on the world-sheet and on the Chan-Paton factors. $\widetilde{O p^{-}}$can be interpreted as the bound state of an $O p^{-}$and half a $D p$ brane, as one obtains the right RR charge for $\frac{1}{2} D p$ on top of an $O p^{-}$. Then adding $n$ more $D p$ 's, we obtain the gauge group $S O(2 n+1)$ which extends the $S O(2 n)$ realization. The transverse space to the orientifolds contains a $R P^{2}$ (resp. $R P^{5-p}$ ) cycle through which the fluxes

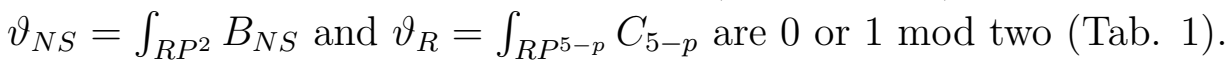

The effective world-volume actions of the $O p^{-}, O p^{+}$planes and $D p$-branes can be determined perturbatively by a string computation [3, 目, [] or by requiring the consistent cancellation of anomalies that arise when pairs of branes intersect [6, []. The perturbative formulae for the orientifold planes and the $D p$-branes are:

$$
\begin{aligned}
W Z\left(O p^{-}\right) & =-2^{p-5} \int_{M_{p+1}} C \wedge \sqrt{\hat{L}(R / 4)} \\
W Z\left(O p^{+}\right) & =2^{p-5} \int_{M_{p+1}} C \wedge(2 \sqrt{\hat{A}(R)}-\sqrt{\hat{L}(R / 4)}) \\
W Z(D p) & =\int_{M_{p+1}} C \wedge \operatorname{ch}(E) \wedge \sqrt{\hat{A}(R)}
\end{aligned}
$$

where $C$ is the sum of RR forms $\sum_{i} C_{i}$. $\hat{A}(R)$ and $\hat{L}(R)$ are respectively the Dirac roof genus and the Hirzebruch polynomial given in terms of Pontryagin classes by:

$$
\begin{aligned}
\hat{A}(R) & =1-\frac{1}{24} p_{1}+\frac{1}{5760}\left(7 p_{1}^{2}-4 p_{2}\right) \\
\hat{L}(R / 4) & =1+\frac{1}{48} p_{1}-\frac{1}{11520}\left(p_{1}^{2}-7 p_{2}\right)
\end{aligned}
$$

The Chern Character of the D-brane bundle appears too and the maximal dimension possible is $p+1=10$. Let us notice that the difference (1.1)-(1.2) only depends on the square root of the roof genus whereas the first topological term (1.1) depends only on the square root of the signature. These perturbative results are strikingly simple.

As we know the transformation of $B_{N S}$ and $C_{2}$ under $S L(2, \mathbb{Z})$, we may deduce the congruence subgroups $\mathcal{G}$ of $S l(2, \mathbb{Z})$ that preserve the various orientifold 3-planes (Tab. 1). It should be stressed that the charges $\left(\vartheta_{N S}, \vartheta_{R}\right)$ transform cogradiently to the gauge fields $\left(B_{N S}, C_{2}\right)$ under $\mathcal{G}$.

In [8], the gravitational coupling of an $\widetilde{O p^{-}}$orientifold was computed by adding the Wess-Zumino terms of an $O p^{-}$orientifold and half a $D p$ brane. The latter was assumed to be one half the Wess-Zumino action of a full $D p$ brane. This hypoth- 
esis seems too strong because the $\frac{D p}{2}$ brane has to be non perturbative to make a perturbative $O p^{-}$into a non perturbative $\widetilde{O p^{-}}$.

We will see in section two that this hypothesis is incorrect. We will compute gravitational couplings for $\widetilde{O p^{-}}$and $\widetilde{O p^{+}}$orientifolds that differ from those of [\&]. To obtain the Wess-Zumino actions of $\widetilde{O p^{-}}$and $\widetilde{O p^{+}}$, we will use the various Sdualities that exist for $p=3$. T-dualities reduce the problem to $p=3$, one then uses non perturbative gravitational couplings for $D 3$, heterotic string and CHL string on $T^{6}$ (in fact perturbative computation and $S L(2, \mathbb{Z})$ invariance lead to the same result up to normalisation for $D 3$ and heterotic string on $T^{6}$ ) and computes those of $\mathrm{O}^{-}$and $\mathrm{O3}^{+}$. One finally gets $\widetilde{\mathrm{O3}^{-}}$and $\widetilde{\mathrm{O3}^{+}}$planes by applying respectively $S$ and $S T$ transformation to $O 3^{+}$. We recall that an orientifold $O(p+1)^{-}$(resp. $\left.O(p+1)^{+}\right)$gives by T-duality along it two $O p^{-}\left(\right.$resp. two $\left.O p^{+}\right)$and an orientifold $O(p+1)^{-}\left(\operatorname{resp} . O(\overline{P+1})^{+}\right)$gives one $\widetilde{O p^{-}}\left(\operatorname{resp} . \widetilde{O p^{+}}\right)$and one $O p^{-}\left(\operatorname{resp} . O p^{+}\right)$ [9]. Flux conservation and dualities relate the $R-R$ charges of the orientifolds. By a perturbative computation, we know that $O 3^{+}$has charge $\frac{1}{4}$. As the $C_{4}$ form is invariant under $S L(2, \mathbb{Z})$, we obtain that the $C_{4}$-charges for $\widetilde{O 3^{-}}$and $\widetilde{O 3^{+}}$are both $\frac{1}{4}$. Then by using T-duality we obtain that the charge of $\widetilde{O p^{-}}$(resp. $\widetilde{O p^{+}}$) is $-2^{p-5}+\frac{1}{2}$ (resp. $\left.2^{p-5}\right)$.

In section three, we relate these results to semi-classical computations in $\mathcal{M}$ theory. They rely crucially on the $M 5$ brane, on the "orientifold" $\mathcal{O M} 5$ and on the ( $x^{11}$ delocalized) lift of $\widetilde{O 4^{-}}[10]$.

\begin{tabular}{|c|c|c|c|c|}
\hline Notation & $\left(\vartheta_{N S}, \vartheta_{R}\right)$ & charge $Q_{p}$ & Group & Self-duality $p=3 \mathcal{G}$ \\
\hline$O p^{-}$ & $(0,0)$ mod. 2 & $-2^{p-5}+n$ & $S O(2 n)$ & $S L(2, \mathbb{Z})$ \\
\hline$O p^{+}$ & $(1,0)$ mod. 2 & $2^{p-5}+n$ & $S p(n)$ & $\Gamma_{0}(2)=\left\{\left(\begin{array}{ll}1 & \star \\
0 & 1\end{array}\right) \bmod 2\right\}$ \\
\hline$\widetilde{O p^{-}}$ & $(0,1) \bmod .2$ & $-2^{p-5}+\frac{1}{2}+n$ & $S O(2 n+1)$ & $\Gamma^{0}(2)=\left\{\left(\begin{array}{ll}1 & 0 \\
\star & 1\end{array}\right) \bmod 2\right\}$ \\
\hline$\widetilde{O p^{+}}$ & $(1,1) \bmod .2$ & $2^{p-5}+n$ & $S p(n)$ & $\Gamma(2)=\left\{\left(\begin{array}{ll}1 & 0 \\
0 & 1\end{array}\right)\right.$ or $\left.\left(\begin{array}{ll}0 & 1 \\
1 & 0\end{array}\right) \bmod 2\right\}$ \\
\hline$n D p$ & & $n$ & $U(n)$ & $S L(2, \mathbb{Z})$ \\
\hline
\end{tabular}

Table 1: Orientifold $p$-planes with $n D p$ branes. $\star$ stands for 0 or 1 .

\section{Dualities}

The Wess-Zumino actions written above can receive nonperturbative corrections coming from $D_{-1}$ instantons stuck on the odd-planes. As the force between $D_{-1}$ and $D_{p}$ branes is not repulsive for $\mathrm{p}=3$ and $\mathrm{p}=7,3$-planes and 7 -planes may receive corrections. Moreover, only the terms with at least $\frac{\mathcal{N}}{4}$ derivatives receive D-instanton 
corrections in the theories with $\mathcal{N}$ SUSY. Indeed, a term with $\frac{n_{f}}{2}$ derivatives is transformed by supersymmetry in a vertex with at most $n_{f}$ fermions. As the $D_{-1}$ instanton leaves half of the $\mathcal{N}$ SUSY unbroken, we have $\frac{\mathcal{N}}{2}$ fermionic modes. To obtain a result different from zero when we integrate over the fermionic coordinates we have the semi-classical rule: $n_{f} \geq \frac{\mathcal{N}}{2}$. In the following section, we will consider theories with $\mathcal{N}=16$. The gravitational couplings with at least 4 derivatives will receive D-instanton corrections. For example, for the orientifold three-planes, only the "BPS-saturated" coupling $\int C_{0} p_{1}$ receives corrections from D-instantons.

We begin with the $S O(32)$ heterotic string compactified on a two torus of Kähler class $T_{H}=B_{N S}+i V$ and complex structure $U_{H}$ at the $S O(8)^{4}$ point corresponding to two Wilson lines $Y_{1}=\left(0^{4}, 0^{4}, \frac{1}{2}^{4}, \frac{1}{2}^{4}\right)$ and $Y_{2}=\left(0^{4}, \frac{1}{2}^{4}, 0^{4}, \frac{1}{2}^{4}\right)$. We first S-dualize to obtain type I and then apply a double T-duality along $T^{2}$. We obtain an orientifold from $I I B$ theory on $T^{2} /\left(\mathbb{Z}_{2}(-)^{F_{L}} \Omega\right)$ with 16 D7's and $4 O 7^{-}$'s where $\mathbb{Z}_{2}$ acts as inversion on the two torus $T^{2}, F_{L}$ is the left moving fermion number and $\Omega$ is the worldsheet parity operator. The moduli $\tau=C_{0}+i e^{-\phi}$ corresponding to the $D_{-1}$ action and $U_{I I B}$ are identified respectively with $T_{H}$ and $U_{H}$. This is F-theory on a special $K 3$ surface (an elliptic fibration with base $T^{2} / \mathbb{Z}_{2}$ [11]). Each of the four singular fibers corresponds to $4 \mathrm{D} 7$ plus one orientifold $O 7^{-}$composed of a $(1,1)$ 7 -plane and a $(1,-1) 7$-plane. We recall that a $(p, q) 7$-plane is a 7 -brane on which a $(p, q)$ string can end [12]. We prove in appendix B, that the action of an $O 7^{-}$ orientifold cannot be obtained by adding the Wess-Zumino terms of a $(1,1)$ and $(1,-$ 1) 7-planes. This is an important obstacle to deriving effective actions, we shall circumvent it by using S-duality in four dimensions.

Let us now compactify further the preceding configuration on a four torus $T^{4}$ and apply four T-dualities, we obtain type IIB theory compactified on a $T^{6} / \mathbb{Z}_{2^{-}}$ orientifold with $16 \mathrm{D} 3^{\text {'s }}$ and $64 \mathrm{O3}^{-}$'s which is dual to the heterotic string on $T^{6}$. We should also note that heterotic string theory on $T^{6}$ is dual to type IIA theory compactified on $K 3 \times T^{2}$. The modulus $T_{H}$ is identified with the Kähler modulus $T_{I I A}$. The term $\int f_{I I A}\left(T_{I I A}\right) p_{1}$ in type IIA theory can be computed exactly from a one-loop calculation for this geometry assuming no purely non perturbative cusp terms appear. Using the duality chain we identify this term with the Wess-Zumino action for $16 \mathrm{D} 3$ and $64 \mathrm{O3}^{-}$. Finally, we have

$$
\int\left(16 f_{D 3}(\tau)+64 f_{03^{-}}(\tau)\right) p_{1}=\int f_{H E T}(\tau) p_{1}=\int \frac{6}{\pi} \operatorname{Re}[i \ln (\eta(\tau))] p_{1}
$$

where $\eta(\tau)$ is the famous Dedekind function and Re is the real part. The properties of the theta functions that we will use in this paper, have been collected in appendix A. The first term on the left handside is a sum because the two terms correspond to the same monodromy.

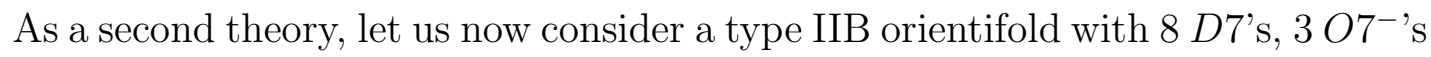
and one $O 7^{+}$. It is T-dual to type IIB on $T^{2}$ modded out by the worldsheet projection 
$\Omega$ in the presence of a half-integral background flux of $B_{N S}$. This orientifold is equivalent to a compactification without vector structure [27, 14] and it is dual to the 8-dimensional CHL string [15, 16]. By compactifying on $T^{4}$ and applying again four T-dualities along it we obtain that the type IIB orientifold on $T^{6} / \mathbb{Z}_{2}$ with 8 $D 3$ 's, $48 \mathrm{O3}^{-}$'s and $16 \mathrm{O3}^{+}$'s is dual to the CHL string on $T^{6}$. In [17], the term $\int f_{C H L}\left(T_{H}\right) p_{1}$ has been computed exactly so we obtain

$$
\int\left(8 f_{D 3}(\tau)+48 f_{03^{-}}(\tau)+16 f_{03^{+}}(\tau)\right) p_{1}=\int f_{C H L}(\tau) p_{1}=\int \frac{1}{\pi} \operatorname{Re}\left[i \ln \left(\vartheta_{2}(\tau) \eta^{3}(\tau)\right)\right] p_{1}
$$

Finally a IIB orientifold with $2 \mathrm{O}^{-}$'s and $2 \mathrm{O}^{+}$'s is S-dual to an asymmetric $\mathbb{Z}_{2}$ orbifold of IIB on $T^{2}$ where the $\mathbb{Z}_{2}$ acts as $(-)^{F_{L}}$ together with a translation on $T^{2}$. There is no more D-brane. We can again compactify on $T^{4}$ and T-dualize. We obtain that IIB orientifold with $32 \mathrm{O3}^{-}$'s and $32 \mathrm{O3}^{+}$'s is dual to an asymmetric $\mathbb{Z}_{2}$ orbifold of IIB on $T^{6}$. This model was argued to be U-dual to type IIA over $\frac{T^{4}}{\mathbb{Z}_{2}} \times T^{2}$ compactification [18]. The $\mathbb{Z}_{2}$ acts both as a twist on the $T^{4}$ and as a shift on the two torus. The modulus $\tau$ is again identified with the Kähler modulus $T_{I I A}$. The coefficient of $p_{1}$ has been computed exactly in type IIA. With the duality chains, we obtain that

$$
\int\left(32 f_{03^{-}}(\tau)+32 f_{03^{+}}(\tau)\right) p_{1}=\int f_{I I B_{\text {asym }}}(\tau) p_{1}=\int \frac{2}{\pi} \operatorname{Re}\left[i \ln \left(\vartheta_{2}(\tau)\right)\right] p_{1}
$$

We now put together our three results: (2.1), (2.2), (2.3), and we obtain the following system for the gravitational couplings of the orientifold three-planes.

$$
\left(\begin{array}{lll}
16 & 64 & 0 \\
8 & 48 & 16 \\
0 & 32 & 32
\end{array}\right)\left(\begin{array}{l}
f_{D 3}(\tau) \\
f_{O 3^{-}}(\tau) \\
f_{O 3^{+}}(\tau)
\end{array}\right)=\left(\begin{array}{l}
\frac{6}{\pi} \operatorname{Re}[i \ln (\eta(\tau))] \\
\frac{1}{\pi} \operatorname{Re}\left[i \ln \left(\vartheta_{2}(\tau) \eta^{3}(\tau)\right)\right] \\
\frac{2}{\pi} \operatorname{Re}\left[i \ln \left(\vartheta_{2}(\tau)\right)\right]
\end{array}\right),
$$

The system (2.4) is singular, in fact the tadpole cancellation condition for the $C_{4}$ charges $Q_{3}$ leads to the constraint $2 f_{C H L}(\tau)=f_{H E T}(\tau)+f_{I I B_{a s y m}}(\tau)$ which is satisfied by our expressions. This is a non trivial check on the various dualities we have used. Now, the $p_{1}$ term for the $D 3$ has already been computed [19, 20] (by S-duality):

$$
f_{D 3}(\tau)=\frac{1}{4 \pi} \operatorname{Re}[i \ln (\eta(\tau))]
$$

So, we solve the independent equations (2.4) and get

$$
\begin{aligned}
& f_{O^{-}}(\tau)=\frac{1}{32 \pi} \operatorname{Re}[i \ln (\eta(\tau))] \\
& f_{\mathrm{O}^{+}}(\tau)=-\frac{1}{32 \pi} \operatorname{Re}\left[i \ln \left(\frac{\eta(\tau)}{\vartheta_{2}^{2}(\tau)}\right)\right]
\end{aligned}
$$


By applying a transformation $S$ or $S T$ on $O 3^{+}$, we obtain respectively the couplings for the $\widetilde{O^{-}}$and $\widetilde{O 3^{+}}$planes:

$$
\begin{aligned}
& f_{\widetilde{O 3^{-}}}(\tau)=-\frac{1}{32 \pi} \operatorname{Re}\left[i \ln \left(\frac{\eta(\tau)}{\vartheta_{4}^{2}(\tau)}\right)\right] \\
& f_{\widetilde{O 3^{+}}}(\tau)=-\frac{1}{32 \pi} \operatorname{Re}\left[i \ln \left(\frac{\eta(\tau)}{\vartheta_{3}^{2}(\tau)}\right)\right]
\end{aligned}
$$

The expressions (2.6), (2.7) in the weak coupling regime $e^{-\phi} \rightarrow \infty$ reproduce the known tree level coupling $p_{1}$. The exponential terms $\sum_{n} \mu(n) e^{2 i \pi n \tau}$ are identified with the $D_{-1}$ contributions. The expressions (2.6), (2.7), (2.8), (2.9) are also invariant under the right duality groups. Indeed, the groups $S L(2, \mathbb{Z}), \Gamma^{0}(2), \Gamma_{0}(2)$ and $\Gamma(2)$ correspond to the invariance groups (modulo phases and $\ln \left(\frac{\tau}{\bar{\tau}}\right)$ terms) of $\eta, \vartheta_{4}, \vartheta_{2}$, $\vartheta_{3}$ respectively.

Moreover, expanding $f_{\widetilde{\mathrm{O3}^{-}}}(\tau)$ and $f_{\widetilde{\mathrm{O3}^{+}}}(\tau)$ in the weak coupling regime, we find

$$
\begin{aligned}
& f_{\widetilde{O 3^{-}}}(\tau)=\frac{1}{384} C_{0}+O\left(e^{2 i \pi n \tau}\right) \\
& f_{\widetilde{O 3^{+}}}(\tau)=\frac{1}{384} C_{0}+O\left(e^{2 i \pi n \tau}\right)
\end{aligned}
$$

By applying T-duality on $\widetilde{\mathrm{O3}^{-}}$and $\widetilde{\mathrm{O3}^{+}}$, we obtain the Wess-Zumino terms for all $p \leq 5$

$$
\begin{aligned}
& W Z\left(\widetilde{O p^{-}}\right)=\left(-2^{p-5}+\frac{1}{2}\right) \int_{M_{p+1}} C \wedge \sqrt{\hat{L}(R / 4)} \\
& W Z\left(\widetilde{O p^{+}}\right)=\int_{M_{p+1}} C \wedge\left(\left(-2^{p-5}+\frac{1}{2}\right) \sqrt{\hat{L}(R / 4)}+\sqrt{\hat{A}(R)}\left(2^{p-4}-\frac{1}{2}\right)\right)
\end{aligned}
$$

We could define the Wess-Zumino action for a half $D p$ brane as the difference between $W Z\left(\widetilde{O p^{-}}\right)$and $W Z(\widetilde{O p})$. We obtain for $p \leq 5$

$$
W Z\left(\frac{D p}{2}\right)=\frac{1}{2} \int_{M_{p+1}} C \wedge \sqrt{\hat{L}(R / 4)}
$$

These results differ from [8] and this can be traced back to the fact that $W Z\left(\widetilde{O 4^{-}}\right)$ is different from zero in there. Our results will be reproduced in the next section through computations in $\mathcal{M}$-theory. Note also that the difference (2.13)-(2.12) depends only on the square root of the roof genus as was the case for the difference (1.1)-(1.2).

\section{M-theory interpretation of Orientifold Planes}

We intend to compare these results with graviton scattering in eleven dimensional supergravity at one-loop. But, first, let us review the $\mathcal{M}$-theory interpretation of 
orientifold four planes [21, 10, 2]. We shall denote by $S_{n}$ a circle along the $\mathrm{n}^{\text {th }}$ direction.

a) $O 4^{-}$is lifted to $\mathcal{M}$-theory on $\mathbb{R}^{4,1} \times \frac{\mathbb{R}^{5}}{\mathbb{Z}_{2}} \times S_{11}$ with $\mathbb{Z}_{2}$ the $\mathbb{R}^{5}$ parity. The fixed plane $\frac{\mathbb{R}^{5}}{\mathbb{Z}_{2}}$ called $\mathcal{O} \mathcal{M} 5$ must carry $-\frac{1}{2}$ the unit charge of an $M 5$ brane in order to cancel the $6 d$ gravitational anomaly [22]. We do not need to add a twisted sector on this plane. Thus, this object carries the same charge as the $O 4^{-}\left(Q_{4}=-\frac{1}{2}\right)$. Compactifying $\mathrm{O4}^{-}$on $S_{9}$ and applying a T-duality along this direction, we obtain two $O 3^{-}$'s in type $I I B$. Now this configuration is lifted to $\mathbb{R}^{3,1} \times \frac{\mathbb{R}^{5}}{\mathbb{Z}_{2}} \times T^{2}$ with the two-torus $T^{2}$ along the nine and eleven directions. The complex IIB coupling $\tau$ is identified with the complex structure $U=\frac{R_{9}}{R_{11}} e^{i \theta}$ of the two-torus $T^{2}$ where $\theta$ is the angle of the torus whose cycles have length $2 \pi R_{9}$ and $2 \pi R_{11}$. Thus, according to (2.6), $\mathcal{O} \mathcal{M} 5$ wrapped on $T^{2}$ should have a term $\int_{\mathbb{R}^{3,1}} \frac{1}{16 \pi} \operatorname{Re}[i \ln (\eta(\tau))] p_{1}$.

b) $O 4^{+}$is lifted to $\mathcal{M}$-theory on $\mathbb{R}^{4,1} \times \frac{\mathbb{R}^{5}}{\mathbb{Z}_{2}} \times S_{11}$ with a $M 5$ brane. This $M 5$ is stuck on the fixed plane of $\mathbb{Z}_{2}$ by imposing as holonomy along $S_{11}$ an element of $O(2)$, $W=\left(\begin{array}{ll}1 & 0 \\ 0 & -1\end{array}\right)$ which is not connected to the identity [10].

This configuration reproduces the charges of the $\mathrm{O4}^{+}$. Compactifying $\mathrm{O4}^{+}$on $S_{9}$ and applying a T-duality, we obtain that a IIB configuration with $2 \mathrm{O}^{+}$is lifted to an $\mathcal{O M} 5$ and a $M 5$ brane both stuck at the fixed point of $\mathbb{Z}_{2}$ and wrapped on $T^{2}$ with a Wilson line $W$. We can deduce from (2.7)-(2.6) that the contribution of the M5 alone must be equal to the difference

$$
2 W Z\left(O 3^{+}-O 3^{-}\right)=\int_{\mathbb{R}^{3,1}} \frac{1}{4 \pi} \operatorname{Re}\left[i \ln \left(\frac{\eta(2 \tau)}{\eta(\tau)}\right)\right] p_{1}
$$

c) $\widetilde{O 4^{-}}$is lifted to $\mathcal{M}$-theory on $\mathbb{R}^{4,1} \times \frac{\mathbb{R}^{5} \times S_{11}}{\mathbb{Z}_{2}}$ with $\mathbb{Z}_{2}$ now the $\mathbb{R}^{5}$ parity times the action on the circle $S_{11}$ by a shift: $x_{11} \rightarrow x_{11}+\pi$. As there is no fixed point, there is no $\mathcal{O M} 5$ and no $M 5$. Moreover, the shift produces a non-trivial "discrete torsion" for the RR 1-form $C_{1}$ in type IIA [10, 21]. So, this configuration reproduces the fact that $\widetilde{O 4^{-}}$has a vanishing $Q_{4}$ charge and a non-trivial RR flux for $\mathcal{C}_{1}$. Compactifying on $S_{9}$ and applying a T-duality, we obtain one $\mathrm{O3}^{-}$and one $\widetilde{\mathrm{O3}^{-}}$. So, M-theory on $\mathbb{R}^{3,1} \times \frac{\mathbb{R}^{5} \times S_{11}}{\mathbb{Z}_{2}} \times S_{9}$ should have a term $\frac{1}{16 \pi} \operatorname{Re}\left[i \ln \left(\vartheta_{4}(\tau)\right)\right] p_{1}$ according to (2.6) and (2.8).

d) Finally, $\widetilde{\mathrm{O4}^{+}}$wrapped on $S_{9}$ (which has also a non trivial discrete torsion for the 1 -form $\mathrm{RR}$ in type $I I A$ ) is lifted to $\mathcal{M}$-theory on $\mathbb{R}^{3,1} \times \frac{\mathbb{R}^{5} \times S_{11}}{\mathbb{Z}_{2}} \times S_{9}$ with a $M 5$ wrapped on $S_{9}$ and stuck at the origin on the circle of half radius $\frac{S_{11}}{\mathbb{Z}_{2}}$. Applying a T-duality along $S_{9}$, we obtain that a pair of $\widetilde{O 3^{+}}$and $O 3^{+}$orientifolds are lifted to $\mathcal{M}$-theory on $\mathbb{R}^{3,1} \times \frac{R^{5} \times T_{911}^{2}}{\mathbb{Z}_{2}}$ with a $M 5$. To be compatible with our previous results, the $M 5$, in this new geometry, must have a term

$$
W Z\left(\widetilde{O 3^{+}}+O 3^{+}-\widetilde{O 3^{-}}-O 3^{-}\right)=\int_{\mathbb{R}^{3,1}} \frac{1}{4 \pi} R e\left[i \ln \left(\frac{\eta(\tau)}{\eta\left(\frac{\tau}{2}\right)}\right)\right] p_{1}
$$


Now we would like to reproduce the terms (3.1), (3.2) which are the $\mathcal{M}$-theory consequences of (2.5), (2.6), (2.7), (2.8), (2.9) from a one- loop computation in $\mathcal{M}$ theory. The topological origin in $\mathcal{M}$-theory of gravitational couplings $\int C_{1} p_{1}$ for four planes is not clear. It was argued in [23] that the chirality of $M 5$ and $\mathcal{O M} 5$ gives the right Wess-Zumino actions for the $\mathrm{D} 4$ brane, orientifolds $\mathrm{O4}^{-}$and $\mathrm{O4}^{+}$, after compactifying on a circle. As the orientifold $\widetilde{O 4^{-}}$is not associated to any $M 5$ or $\mathcal{O M} 5$ in $\mathcal{M}$-theory, it is quite encouraging to find that its Wess-Zumino action vanishes. Although we do not know how to reproduce the terms (3.1), (3.2) for the $M 5$ brane, we are going to reproduce terms proportional to $\operatorname{tr} R \wedge \star R$. As we have at least $\mathcal{N}=1$ in $d=4$, the CP-even and odd parts $A_{\text {even }}$ and $A_{\text {odd }}$ are the same up to a factor $i$ [24].

A M5 wrapped on a two torus $T^{2}$ with a Wilson line $W$ must have a term

$$
\int_{\mathbb{R}^{3,1}} \frac{1}{4 \pi} \operatorname{Re}\left[\ln \left(\frac{\eta(\tau)}{\eta(2 \tau)}\right)\right] \operatorname{tr} R \wedge \star R
$$

Moreover a M5 wrapped on $S_{9}$ and stuck on a circle of half radius reproduces the term

$$
\int_{\mathbb{R}^{3,1}} \frac{1}{4 \pi} \operatorname{Re}\left[\ln \left(\frac{\eta\left(\frac{\tau}{2}\right)}{\eta(\tau)}\right)\right] \operatorname{tr} R \wedge \star R
$$

Let us now consider a one-loop diagram with two external gravitons scattering in the world volume of a M5 brane (Fig. 1).

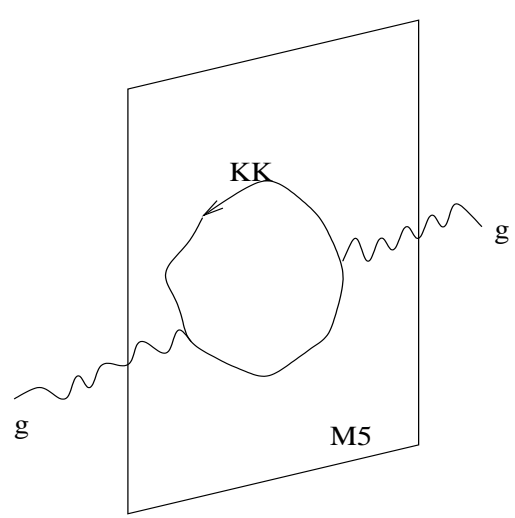

Figure 1: Two external gravitons scattering in the world volume of a $M 5$ brane.

This one-loop effect can be computed as the sum of Feynman diagrams with the $(2,0)$ tensor multiplet of the $M 5$ circulating in the loop. The Kaluza-Klein modes with momenta $p_{11}=\frac{l_{11}}{R_{11}}$ and $p_{9}=\frac{l_{9}}{R_{9}}$ circulating in the loop will be interpreted as a bound state of $l_{11}$ D0 branes that moves along an euclidean times $x^{9}$ in type $I I A$ and as all $\left(l_{9}, l_{11}\right)$ D-instantons in $I I B$ theory on the orientifold plane. This 
loop amplitude can be also calculated by using the light cone description of the superparticle [25].

The expression for the one-loop amplitude is [25], [20]:

$$
A_{\text {even }}=\int_{\mathbb{R}^{3,1}} \frac{1}{16 \pi} \operatorname{tr} R \wedge \star R \int_{0}^{\infty} \frac{d t}{t} \sum_{l_{I}} e^{-\pi t G^{I J} l_{I} l_{J}}
$$

where $G^{I J} l_{I} l_{J}=\frac{\left|l_{9}-l_{11} \tau\right|^{2}}{V_{2} \tau_{2}}$. The overall normalization is chosen in order to reproduce (2.5) the correction for a D3 brane [20]. A T-dualized D3 is lifted to a M5 wrapped on a two torus, in this case $l_{I} \in \mathbb{Z}^{2}$. Finally

$$
A_{\text {even }}=\int_{\mathbb{R}^{3,1}} \frac{1}{16 \pi} \operatorname{tr} R \wedge \star R \int_{0}^{\infty} \frac{d t}{t} \sum_{l_{I} \in \mathbb{Z}^{2}} e^{-\pi t G^{I J} l_{I} l_{J}}
$$

Doing a Poisson resummation, we obtain

$$
A_{\text {even }}=\int_{\mathbb{R}^{3,1}} \frac{1}{16 \pi^{2}} \operatorname{tr} R \wedge \star R \sum_{w^{I} \in \mathbb{Z}^{2}} \frac{\tau_{2}}{\left|w_{11}-w_{9} \tau\right|^{2}}
$$

This Maass function can be regularized by eliminating the pole at $s=1$ :

$$
\hat{E}_{1}=\lim _{s \rightarrow 1}\left(\sum_{w^{I} \in \mathbb{Z}^{2} \backslash(0,0)}\left(\frac{\tau_{2}}{\left|w_{11}-w_{9} \tau\right|^{2}}\right)^{s}-\frac{\pi}{s-1}\right)=-4 \pi R e[\ln (\eta(\tau))]
$$

modulo a perturbatively invisible logarithmic term $-\pi \ln \left(\tau_{2}\right)$, not present in the Wilsonian effective action of the orientifolds and D3 branes, so we have removed it by hand. So, we obtain

$$
A_{\text {even }}=-\frac{1}{4 \pi} \operatorname{Re}[\ln (\eta(\tau))] \int_{\mathbb{R}^{3,1}} \operatorname{tr} R \wedge \star R
$$

Now, we need to determine properly the Kaluza-Klein modes which are identified with $\left(l_{9}, l_{11}\right)$ D-instantons, ie the ranges of values of $\left(l_{9}, l_{11}\right)$ for the other configurations. We use that the number of bound states for $(0,1)$ instantons on an orientifold $\mathrm{O3}^{-}$or $\widetilde{\mathrm{O3}^{-}}$(resp. $\mathrm{O3}^{+}$or $\widetilde{\mathrm{O3}^{+}}$) is integer (resp. half integer) as the gauge group is $S O$ (resp. $S p$ ). This analysis has been done for the system $(0,1)$ instanton (ie $D_{-1}$ ) on $O 3^{ \pm}$in [26] and for the system $D_{-1}$ on $\widetilde{O 3^{ \pm}}$in [27. This can also be understood in the picture IIA-M $\left[10 \|\right.$. Now, doing a S-duality exchanges $O 3^{+}$with $\widetilde{O 3^{-}}$and permutes $l_{9}$ with $l_{11}$. A transformation $\mathrm{T}$ exchanges $\widetilde{\mathrm{O3}^{-}}$with $\widetilde{\mathrm{O3}^{+}}$and shifts $l_{9}$ by $l_{11}$. So, using the various self-duality subgroups preserving the orientifold 3-planes (see Table 1), we list the number of D-instantons living on them the solution in Table 2 below. 


\begin{tabular}{|c|c|c|c|c|}
\hline$\left(l_{9}, l_{11}\right) \bmod 2$ & $\mathrm{O3}^{-}$ & $\mathrm{O3}^{+}$ & $\widetilde{O 3}^{-}$ & $\widetilde{\mathrm{O3}^{+}}$ \\
\hline$(0,1)$ & 1 & $\frac{1}{2}$ & 1 & $\frac{1}{2}$ \\
\hline$(1,0)$ & 1 & 1 & $\frac{1}{2}$ & $\frac{1}{2}$ \\
\hline$(1,1)$ & 1 & $\frac{1}{2}$ & $\frac{1}{2}$ & 1 \\
\hline
\end{tabular}

Table 2: Minimal topological charges for $\left(l_{9}, l_{11}\right)$ instantons on orientifold three-planes.

Now let us compute in the two cases including an M5 brane. We first want to reproduce the expression for a $M 5$ wrapped on $T^{2}$ with a Wilson line W. The Kaluza-Klein circulating in the loop correspond to instantons stuck on $\mathrm{O3}^{+}$and we need to sum the one-loop amplitude over $\frac{\mathbb{Z}}{2} \cdot(0,1), \mathbb{Z} .(1,0)$ and $\frac{\mathbb{Z}}{2} \cdot(1,0)$.

First, the one-loop amplitude is a sum over $\frac{\mathbb{Z}}{2} \cdot(0,1)$, this means that we must $\operatorname{sum}\left(\right.$ (3.5) over $\left(l_{9} \in \mathbb{Z}, l_{11} \in \mathbb{Z}+\frac{1}{2}\right)$ and $(2 \mathbb{Z}, 2 \mathbb{Z}+1)$ :

$$
A_{\text {even }}^{\frac{\mathbb{Z}}{2} \cdot(0,1)}=\frac{1}{16 \pi} \int_{\mathbb{R}^{3,1}} \operatorname{tr} R \wedge \star R \int_{0}^{\infty} \frac{d t}{t}\left(\sum_{\left(l_{9} \in \mathbb{Z}, l_{11} \in \mathbb{Z}+\frac{1}{2}\right)}+\sum_{(2 \mathbb{Z}, 2 \mathbb{Z}+1)}\right) e^{-\pi t G^{I J} l_{I} l_{J}}
$$

By rescaling the second summation, we obtain

$$
A_{\text {even }}^{\frac{\mathbb{Z}}{2} \cdot(0,1)}=2 \frac{1}{16 \pi} \int_{\mathbb{R}^{3,1}} \operatorname{tr} R \wedge \star R \int_{0}^{\infty} \frac{d t}{t} \sum_{\left(l_{9} \in \mathbb{Z}, l_{11} \in \mathbb{Z}+\frac{1}{2}\right)} e^{-\pi t G^{I J} l_{I} l_{J}}
$$

This can be written as:

$$
A_{\text {even }}^{\frac{\mathbb{Z}}{2} \cdot(0,1)}=2 \frac{1}{16 \pi} \int_{\mathbb{R}^{3,1}} \operatorname{tr} R \wedge \star R \int_{0}^{\infty} \frac{d t}{t}\left(\sum_{\left(l_{9} \in \mathbb{Z}, l_{11} \in \frac{\mathbb{Z}}{2}\right)}-\sum_{(\mathbb{Z}, \mathbb{Z})}\right) e^{-\pi t G^{I J} l_{I} l_{J}}
$$

Using the regularization (3.8), we obtain the result

$$
A_{\text {even }}^{\frac{\pi}{2} \cdot(0,1)}=\int_{\mathbb{R}^{3,1}} \frac{1}{4 \pi} R e\left[\ln \left(\frac{\eta(\tau)}{\theta_{4}(\tau)}\right)\right] \operatorname{tr} R \wedge \star R
$$

By analogy with the previous rule, the one-loop amplitude over $\mathbb{Z}$.(1,0) is

$$
A_{\text {even }}^{\mathbb{Z} .(1,0)}=\frac{1}{16 \pi} \int_{\mathbb{R}^{3,1}} \operatorname{tr} R \wedge \star R \int_{0}^{\infty} \frac{d t}{t} \sum_{\left(l_{9} \in 2 \mathbb{Z}+1, l_{11} \in 2 \mathbb{Z}\right)} e^{-\pi t G^{I J} l_{I} l_{J}}
$$

Doing the same kind of algebraic manipulation as previously, we obtain

$$
A_{\text {even }}^{\mathbb{Z} .(1,0)}=\int_{\mathbb{R}^{3,1}} \frac{1}{8 \pi} R e\left[\ln \left(\frac{\eta(\tau)}{\theta_{2}(\tau)}\right)\right] \operatorname{tr} R \wedge \star R
$$


Moreover, by applying some $S L(2, \mathbb{Z})$ transformations, we can obtain the following one- loop amplitudes:

$$
\begin{aligned}
& A_{\text {even }}^{\frac{\mathbb{Z}}{2}(1,1)}=\int_{\mathbb{R}^{3,1}} \frac{1}{4 \pi} \operatorname{Re}\left[\ln \left(\frac{\eta(\tau)}{\theta_{3}(\tau)}\right)\right] \operatorname{tr} R \wedge \star R \\
& A_{\text {even }}^{\frac{\mathbb{Z}}{2} \cdot(1,0)}=\int_{\mathbb{R}^{3,1}} \frac{1}{8 \pi} \operatorname{Re}\left[\ln \left(\frac{\eta(\tau)}{\theta_{2}(\tau)}\right)\right] \operatorname{tr} R \wedge \star R \\
& A_{\text {even }}^{\mathbb{Z} \cdot(1,1)}=\int_{\mathbb{R}^{3,1}} \frac{1}{8 \pi} \operatorname{Re}\left[\ln \left(\frac{\eta(\tau)}{\theta_{3}(\tau)}\right)\right] \operatorname{tr} R \wedge \star R
\end{aligned}
$$

Finally, the summation over the D-instantons stuck on $\mathrm{O3}^{+}$is

$$
A_{\text {even }}^{\frac{\mathbb{Z}}{2} \cdot(0,1)}+A_{\text {even }}^{\mathbb{Z} \cdot(1,0)}+A_{\text {even }}^{\frac{\mathbb{Z}}{2} \cdot(1,1)}=\int_{\mathbb{R}^{3,1}} \frac{1}{4 \pi} \operatorname{Re}\left[\ln \left(\frac{\eta(\tau)}{\eta(2 \tau)}\right)\right] \operatorname{tr} R \wedge \star R
$$

We obtain the right result (3.3).

Now, we do the same thing for $M 5$ wrapped on $S_{9}$ and stuck on a circle of half radius $S_{11} / 2$. We have seen previously that the Kaluza-Klein modes stuck on $M 5$

correspond to D-instantons on $\mathrm{O}^{+}$and $\widetilde{\mathrm{O3}^{-}}$. So, the one-loop amplitude, according to the table 2, is:

$2 A_{\text {even }}^{\frac{\mathbb{Z}}{2} \cdot(0,1)}+A_{\text {even }}^{\mathbb{Z} \cdot(1,0)}+A_{\text {even }}^{\frac{\mathbb{Z}}{2} \cdot(1,1)}+A_{\text {even }}^{\frac{\mathbb{Z}}{2} \cdot(1,0)}+A_{\text {even }}^{\mathbb{Z} \cdot(1,1)}=\int_{\mathbb{R}^{3,1}} \frac{1}{4 \pi} \operatorname{Re}\left[\ln \left(\frac{\eta\left(\frac{\tau}{2}\right)}{\eta(\tau)}\right)\right] \operatorname{tr} R \wedge \star R$

We obtain the right result (3.4)

\section{Conclusion:}

Using various string dualities, we have found the Wess-Zumino actions for orientifold planes with a non trivial $R R$ flux. The $p=7$ analog problem remains open. The precise rules of instantonic computation are not known but this could be circumvented by using $\mathrm{S}$, $\mathrm{T}$ dualities. We have used (following [10]) the lift of orientifolds $\widetilde{O p^{ \pm}}$in $\mathcal{M}$ theory without giving a name as they are not localized. But we found a set of rules for D-instantons living on orientifold three planes.

\section{Acknowledgments}

We have benefited from discussions with C. Bachas, P. Bain, A. Hanany, E. Kiritsis, L. Paulot, P. Vanhove and especially A. Keurentjes.

\section{Appendix A: Properties of Theta functions}


we set $q=e^{2 i \pi \tau}$

$$
\begin{aligned}
\eta(\tau) & =q^{\frac{1}{24}} \prod_{n=1}^{\infty}\left(1-q^{n}\right) \\
\vartheta_{2}(\tau) & =2 q^{\frac{1}{8}} \prod_{n=1}^{\infty}\left(1-q^{n}\right)\left(1+q^{n}\right)^{2}=2 \frac{\eta^{2}(2 \tau)}{\eta(\tau)} \\
\vartheta_{3}(\tau) & =\prod_{n=1}^{\infty}\left(1-q^{n}\right)\left(1+q^{n+\frac{1}{2}}\right)^{2}=2 \frac{e^{\frac{-i \pi}{3}}}{1-\tau} \frac{\eta^{2}\left(\frac{1+\tau}{1-\tau}\right)}{\eta(\tau)} \\
\vartheta_{4}(\tau) & =\prod_{n=1}^{\infty}\left(1-q^{n}\right)\left(1-q^{n-\frac{1}{2}}\right)^{2}=\frac{\eta^{2}\left(\frac{\tau}{2}\right)}{\eta(\tau)} \\
2 \eta^{3}(\tau) & =\vartheta_{2} \vartheta_{3} \vartheta_{4}
\end{aligned}
$$

\begin{tabular}{|c|c|c|}
\hline & $\mathrm{S}$ & $\mathrm{T}$ \\
\hline$\eta(\tau)$ & $\sqrt{-i \tau} \eta(\tau)$ & $e^{\frac{2 \pi}{12}} \eta(\tau)$ \\
\hline$\vartheta_{2}(\tau)$ & $\sqrt{-i \tau} \vartheta_{4}(\tau)$ & $e^{\frac{2 \pi}{4}} \vartheta_{2}(\tau)$ \\
\hline$\vartheta_{3}(\tau)$ & $\sqrt{-i \tau} \vartheta_{3}(\tau)$ & $\vartheta_{4}(\tau)$ \\
\hline$\vartheta_{4}(\tau)$ & $\sqrt{-i \tau} \vartheta_{2}(\tau)$ & $\vartheta_{3}(\tau)$ \\
\hline
\end{tabular}

Table 3: Transformations of the Theta functions under the generators $\mathrm{S}$ and $\mathrm{T}$ of $S L(2, \mathbb{Z})$

Poisson's Resummation:

$$
\sum_{\vec{l} \in Z} e^{-\pi(\vec{l}+\vec{x}) \cdot A \cdot(\vec{l}+\vec{x})}=A^{-\frac{1}{2}} \sum_{\vec{w} \in Z} e^{-\pi \vec{w} \cdot A^{-1} \cdot \vec{w}+2 \pi i \vec{w} \cdot \vec{x}}
$$

\section{Appendix B: The puzzle of seven planes}

The Green-Schwarz terms for the Heterotic string compactified on $T^{2}$ (with gauge group $\left.S O(8)^{4}\right)$, dual to IIB orientifold with $16 \mathrm{D} 7$ and $4 \mathrm{Or}^{-}$, has already been computed in 28]. For example, we can deduce that

$$
\int\left(16 f_{D 7}(\tau)+4 f_{O 7^{-}}(\tau)\right) p_{2}=\int \frac{1}{120 \pi} \operatorname{Re}\left[i \ln \left(\vartheta_{4}(\tau) \eta(\tau)\right)\right] p_{2}
$$

Now, we will assume that the Wess-Zumino action of $O 7^{-}$can be obtained by adding the action of a $(1,1)$ and $(1,-1) 7$-planes. We can note that even though these $(p, q)$ 7-planes are associated with strong coupling and may not have a well-defined Lagrangian description, the Wess-Zumino terms are topological and must make sense at least for anomaly cancelling reasons. 
By definition, the action of a (p,q) 7-plane is found by applying the inverse of

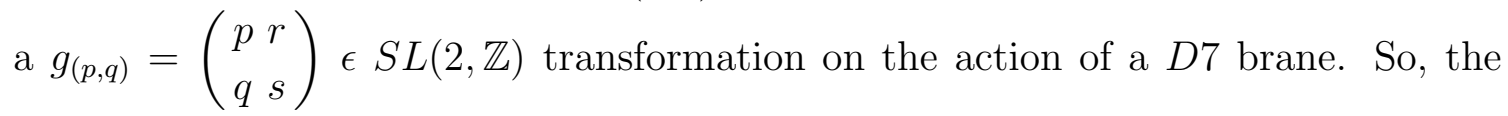
function $f_{D 7}(\tau)$ must be invariant under $\mathrm{T}$ so that the action for the $(\mathrm{p}, \mathrm{q})$ branes does not depend on the integers $\mathrm{r}$ and $\mathrm{s}$. Then, if we ignore possible interaction terms, we obtain:

$$
16 f_{D 7}(\tau)+4\left(f_{D 7}\left(g_{(1,1)}^{-1} \tau\right)+f_{D 7}\left(g_{(1,-1)}^{-1} \tau\right)\right)=\frac{1}{120 \pi} \operatorname{Re}\left[i \ln \left(\vartheta_{4}(\tau) \eta(\tau)\right)\right]
$$

Algebraic manipulations imply that T-invariance of $D 7$ cannot hold.

Thus, some work remains to be done in order to find the non-perturbative Dinstanton corrections for $\mathrm{D} 7, \mathrm{O}^{-}$and $\mathrm{O}^{+}$.

\section{References}

[1] E. Witten, Baryons And Branes In Anti de Sitter Space, JHEP 9807 (1998) 006, hep-th/9805112

[2] A. Hanany, B. Kol, On Orientifolds, Discrete Torsion, Branes and M Theory, JHEP 0006 (2000) 013, hep-th/0003025

[3] B. Craps, F. Roose, Anomalous D-brane and orientifold couplings from the boundary state, Phys.Lett. B445 (1998) 150-159, hep-th/9808074

[4] J.F. Morales, C.A. Scrucca, M. Serone, Anomalous couplings for D-branes and Oplanes, Nucl. Phys. B552 (1999) 291-315, hep-th/9812071

[5] B. Stefanski Jr, Gravitational Couplings of D-branes and O-planes, Nucl. Phys. B548 (1999) 275-290, hep-th/9812088

[6] M. Green, J. A. Harvey, G. Moore, I-Brane Inflow and Anomalous Couplings on DBranes, Class.Quant.Grav. 14 (1997) 47-52, hep-th/9605033

[7] C.A. Scrucca, M. Serone, Anomalies and inflow on D-branes and O-planes, Nucl. Phys. B556 (1999) 197-221, hep-th/9903145

[8] S. Mukhi, N. V. Suryanarayana, Gravitational Couplings, Orientifolds and M-Planes, JHEP 9909 (1999) 017, hep-th/9907215

[9] A. Keurentjes, Classifying orientifolds by flat n-gerbes, hep-th/0106267

[10] E. G. Gimon, On the M-theory Interpretation of Orientifold Planes, hep-th/9806226

[11] A. Sen, F-theory and Orientifolds, Nucl.Phys. B475 (1996) 562-578, hep-th/9605150

[12] J. H. Schwarz, An SL(2,Z) Multiplet of Type IIB Superstrings, Phys.Lett. B360 (1995) 13-18; Erratum-ibid. B364 (1995) 252, hep-th/9508143 
[13] E. Witten, Toroidal Compactification Without Vector Structure, JHEP 9802 (1998) 006, hep-th/9712028

[14] M. Bianchi, A Note on Toroidal Compactifications of the Type I Superstring and Other Superstring Vacuum Configurations with 16 Supercharges Nucl.Phys. B528 (1998) 7394, hep-th/9711201

[15] S. Chaudhuri, G. Hockney, J. D. Lykken, Maximally Supersymmetric String Theories in $D \leq 10$, Phys.Rev.Lett. 75 (1995) 2264-2267, hep-th/9505054

[16] W. Lerche, R. Minasian, C. Schweigert, S. Theisen, A Note on the Geometry of CHL Heterotic Strings, Phys.Lett. B424 (1998) 53-59, hep-th/9711104

[17] A. Gregori, E. Kiritsis, C. Kounnas, N. A. Obers, P. M. Petropoulos, B.Pioline, $R^{2}$ Corrections and Non-perturbative Dualities of $N=4$ String ground states, Nucl.Phys. B510 (1998) 423-476, hep-th/9708062

[18] A. Sen, C. Vafa, Dual Pairs of Type II String Compactification , Nucl.Phys. B455 (1995) 165, hep-th/9508064

[19] K. Dasgupta, D. P. Jatkar, S. Mukhi, Gravitational Couplings and $\mathbb{Z}_{2}$ Orientifolds, Nucl.Phys. B523 (1998) 465-484, hep-th/9707224

[20] C. P. Bachas, P. Bain, M. B. Green, Curvature terms in D-brane actions and their M-theory origin , JHEP 9905 (1999) 011, hep-th/9903210

[21] K. Hori, Consistency Conditions for Fivebrane in $M$ Theory on $R^{5} / \mathbb{Z}_{2}$ Orbifold, Nucl.Phys. B539 (1999) 35-78, hep-th/9805141

[22] E. Witten, Five-branes And M-Theory On An Orbifold, Nucl.Phys. B463 (1996) 383397, hep-th/9512219

[23] S. Mukhi, Dualities and the SL(2,Z) Anomaly, JHEP 9812 (1998) 006, hep-th/9810213

[24] I. Antoniadis, E. Gava, T. R. Taylor, Phys.Lett.B 267 (1991), 37

[25] M.B. Green, M. Gutperle, P. Vanhove, One loop in eleven dimensions, Phys.Lett. B409 (1997) 177-184, hep-th/9706175

[26] E. G. Gimon, J. Polchinski, Consistency Conditions for Orientifolds and D-Manifolds, Phys.Rev. D54 (1996) 1667-1676, hep-th/9601038

[27] Edward Witten, Toroidal Compactification Without Vector Structure, HEP 9802 (1998) 006, hep-th/9712028

[28] E. Kiritsis, N. A. Obers, B. Pioline, Heterotic / Type II Triality and Instantons on K(3),JHEP 0001:029,2000, hep-th/0001083

[29] W. Lerche, On the Heterotic/F-Theory Duality in Eight Dimensions, proceedings of Cargese 1999, hep-th/9910207 